\title{
Thermal and Dynamical Investigation on the Effect of Aminofunctionalized Carbon Nanotubes Embedded in an Epoxy Matrix.
}

\author{
T. V. Kosmidou ${ }^{1,}$ E. Nikolaidou ${ }^{1,2}$, A. Z. Stimoniaris ${ }^{1,2}$, A. S. Vatalis ${ }^{1}$ and C. G. Delides ${ }^{1, *}$ \\ ${ }^{1}$ Technological Educational Institute of Western Macedonia, Laboratories of Physics and Materials Technology, 50100, \\ Kila, Kozani, Greece \\ ${ }^{2}$ Materials Science and Technology Dpt, University of Ioannina
}

Received 24 January 2011; Revised 17 March 2011; Accepted 9 December 2011

\begin{abstract}
In the present work an epoxy/carbon nanotubes composite system is studied with the main focus on the effect of the network architecture on the thermophysical properties of the system. The fillers' modification plays an important role in the formation of a secondary crosslinking network, between the amine groups of the nanotubes and the epoxy rings of the prepolymer. The secondary network seems to affect the final properties of the product. The results indicate the existence of an interfacial layer around the filler particles and the coexistence of two networks. The discussion includes the effect of the filler content, the dispersion conditions and the architectural structure of the secondary network on the $\alpha$-and $\beta$-relaxations corresponding to the associated temperatures Tg and T $\beta$ and the thermodynamic behavior of the produced material.
\end{abstract}

Keywords: Epoxy nanocomposites. Functionalised Carbon Nanotubes, Secondary crosslinking network

\section{Introduction}

Epoxies, the most common thermosets, are widely used and therefore already widely studied. In spite of the fact that they have been widely investigated, there are still some unresolved issues in this area. In the case of utilizing an epoxy system as a matrix component of a composite material, there are a lot of possibilities in choosing the appropriate filler, depending on the final application. The most recent research involves the investigation of nano-sized fillers, and especially carbon nanotubes $[1,2]$.

The properties of a nanocomposite system may vary significantly from those of the bulk matrix because of the small size of the filler and the increase of the interfacial area [3-9]. The interfacial area, that is the area formed between the surface of the filler and the matrix, plays a critical role in the overall behaviour of the system. The properties of this phase depend on the properties of the components and could be "manipulated" according to the requirements of the application. The systems properties can also be affected by the dispersion state, the shape, the surface properties and the size of the filler.

The recent commercial availability of carbon nanotubes has led the researchers to investigate the appropriate modifications in order to increase the compatibility of the nanotubes with the potential matrices. Carbon nanotubes are rod shaped formations either open ended or closed with end caps, with either single graphite wall or multiple graphite walls on the side surface. Taking advantage of their amazing mechanical-thermal-electrical properties in widely used systems is the main request.

For example, when studying tribological behaviour and wear resistance of epoxies, it was found that there is a dramatical improvement when they are reinforced with Multi walled Carbon Nanotubes (MWCNT). The MWCNT's are acting as natural lubricants, as they reduce the friction and improve the wear resistance of the polymer [10].

In the case of mechanical properties of epoxy based composites, it has been reported that when simple fillers are used, without any modification on their surface, there is a decrease in the flexural strength of the system, as the filler content increases. By improving the interface quality, i.e. the adhesive strength and the interfacial stiffness of the system, the mechanical strength increases with the increase of the filler. [11]

The main mechanisms of load transfer from a matrix to a filler[12] is a)micro mechanical interlocking where local non uniformity along a CNT, including varying diameter and bends at places as a result of non-hexagonal defects, contribute to CNT-polymer adhesion by mechanical interlocking, b)chemical bonding between the nanotubes and the matrix, this improves interfacial interactions through ionic or covalent bond that enables stress transfer and c)weak van der Waals bonding between the fibre and the matrix when under no chemical bonding between CNTpolymer, the origins of the CNT-polymer interactions are electrostatic and van der Waals forces. 
T. V. Kosmidou, E. Nikolaidou, A. Z. Stimoniaris, A. S. Vatalis and C. G. Delides/

Journal of Engineering Science and Technology Review 4 (2) (2011) 169-173

Apart from the interfacial layer itself, there is also a need to improve the dispersion morphology of the system. The nanotubes tend to form agglomerates which do not contribute positively to the overall behaviour of a composite. In systems where the dispersion of the tubes was poor, there was also a great percentage of MWCNT's that were pulled out during the fracture of the composite [13]. In the same study, where dielectric measurements were performed in such systems, it was found that with an appropriate modification of the surface of the MWCNT's the dielectric constant increases up to 60 times. The modification in this case was the treatment of the MWCNT's with amine. This treatment doesn't change the graphite structure of the surface, but it assists the matrix-filler adhesion by adding a $\mathrm{C}-\mathrm{N}$ bond and an N-H bond to the interface area. The FTIR spectra of the system, confirms the existence of these bonds and the disappearance of the $\mathrm{NH}_{2}$ groups that were present in the raw materials. [13].

Concerning the thermal behaviour of such systems, it is confirmed that the specific heat increases and the thermal conductivity increases up to $40 \%$ with the use of amino functionalised MWCNT's.

The glass transition temperature appeared to decrease down to $30 \%$ when the nanotubes used were not aminemodified, this fact is justified by the finding that the molecules of the epoxy are of the same order of magnitude with the CNT's and thus the non modified CNT's can act as plasticizers of the polymer[14].

The effect of the nanofillers on the glass transition $\left(\mathrm{T}_{\mathrm{g}}\right)$ and the relaxation behaviour of the polymer matrix has been reported for different composite systems. As mentioned before, in some cases an increase in $\mathrm{T}_{\mathrm{g}}$ was observed [4-6, 9, 14-16] but in others a decrease for a similar system is reported $[7,14]$. An initial decrease in $T_{g}$ followed by a $T_{g}$ increase with a higher filler content was observed in epoxy/carbon nanotubes composites by Berriot et al. [17]. In many studies, the amount, the dispersion and the surface conditions of the nanoparticles play important role in the changes in $T_{g}$ and the mechanical properties of the nanocomposites $[4,8,9,14]$. In the present study there is also a part dedicated to the investigation of the $\beta$-relaxation $\left(T_{\beta}\right)$, which occurs in temperatures well below zero. This low temperature behaviour can be critical in aerospace applications and can lead to a failure of the system, due to differences in thermal expansion coefficients of the components [14].

In the current study using a variety of experimental techniques, the reinforcement of epoxy matrix due to incorporation of amine-functionalised carbon nanotubes is examined. The amine groups on the fillers surface act as an additional curing agent, so that the architecture of the crosslinking network is significantly different from that of the pure matrix or a matrix reinforced with nonfunctionalized carbon nanotubes.

\section{Materials}

The prepolymer D.E.R.332 used in this study was diglycidil ether of bisphenol A (DGEBA) supplied by Fluka SA, USA. The hardener used was triethylenetetramine (TETA) supplied by Sigma Aldrich, USA. The multi-wall carbon nanotubes (NC3152) were supplied by Nanocyl, Belgium. They were produced via the catalytic carbon vapor deposition (CCVD) process. The nanotubes which exited the reactor were first purified and then functionalized via a
Nanocyl patented process with $-\mathrm{NH}_{2}$ groups. Description of the MWCNTs, as given by the manufacturer, is presented in Table 1 .

Table 1: MWCNTs' description

\begin{tabular}{llll}
\hline Property & Unit & Value & $\begin{array}{l}\text { Method Of } \\
\text { Measurement }\end{array}$ \\
\hline Average Diameter & nanometers & 9.5 & TEM \\
Average Length & microns & $<1$ & TEM \\
Carbon Purity & $\%$ & $>95$ & TGA \\
Metal Oxide & $\%$ & $<5$ & TGA \\
$\begin{array}{l}\text { Amorphous } \\
\begin{array}{l}\text { Carbon } \\
\text {-NH2 }\end{array}\end{array}$ & - & $*$ & HRTEM \\
$\begin{array}{l}\text { Functionalization } \\
\text { (NC3152) }\end{array}$ & & $<0.5$ & XPS \\
& & & \\
\hline
\end{tabular}

* Pyrolytically deposited carbon on the surface of the CNT

\section{Sample preparation}

The DGEBA/TETA/MWCNTs nanocomposites were prepared by sonication in order to accomplish the best possible dispersion of the carbon nanotubes in the matrix of the epoxy resin. The process involves six steps.

- $\quad$ First the MWCNTs are added to the prepolymer (DGEBA) and the mixture is mechanically stirred for 30 minutes.

- $\quad$ After the first step, the DGEBA/MWCNTs mixture is sonicated for 30 minutes at $105 \mathrm{~W}$.

- Then the appropriate amount of hardener is added and the mixture is mechanically stirred for 20 minutes. The amount of hardener was chosen to be the stoichiometric amount of $14 \mathrm{pphr}$ (parts per hundred resin).

- The final mixture is placed 30 minutes in vacuum for degassing.

- The mixture is poured in teflon moulds.

And the last two steps involve.

- Curing of the samples at $60{ }^{\circ} \mathrm{C}$ for 2 hours.

- Post curing at $160{ }^{\circ} \mathrm{C}$ for second session of 2 hours.

\section{Methods}

\section{Differential Scanning Calorimetry (DSC)}

The measurements were performed with a Perkin Elmer device in a range from room temperature up to $200{ }^{\circ} \mathrm{C}$ at a programmed heating rate of $20^{\circ} \mathrm{C} /$ min under $\mathrm{N}_{2}$ atmosphere.

Thermogravimetric Analysis (TGA)

A Perkin Elmer analyzer was used at the same heating rate of $2^{0} \mathrm{C} / \mathrm{min}$, under $\mathrm{N}_{2}$ atmosphere.

Dynamic Mechanical thermal Analysis (DMA)

A Polymer Laboratories dynamic mechanical thermal analyzer PL MK II was used. Measurements were performed from room temperature up to $200^{\circ} \mathrm{C}$ on a constant rate of $2^{0} \mathrm{C} / \mathrm{min}$, the frequency was set at $10 \mathrm{~Hz}$, the strain at $4 \mathrm{x}$ and the resultant changes in $\mathrm{E}^{\prime}$ and $\tan \delta$ were plotted. 


\section{Results and discussion}

To date, there has been no apparent consensus on the thermomechanical properties for epoxy/CNTs.nanocomposites. For example, the values of $\mathrm{T}_{\mathrm{g}}$ reported in the literature for typical epoxy/ CNTs nanocomposites vary from $66{ }^{\circ} \mathrm{C}$ to $155^{\circ} \mathrm{C}$ [1-9] depending on the modification of CNTs and the processing techniques used to produce the nanocomposites. The wide variation in the $T_{g}$ values indicates that the dispersion states and other properties such as bundle formation, agglomeration and/or aggregation of CNTs affected by different processing conditions are important in determining the thermomechanical properties of epoxy-CNT nanocomposites. Therefore, it is very interesting to study the network structure after the introduction of aminofunctionalized MWCNTs into the matrix for accessing their reinforcement role.

The use of modified MWCNTs introduces new aspects to the architecture of the composites formed. The fillers' modification allows a better dispersion into the matrix, but it also plays an important role to the formation of a secondary crosslinking network, between the amine groups of the nanotubes and the epoxy rings of the prepolymer.

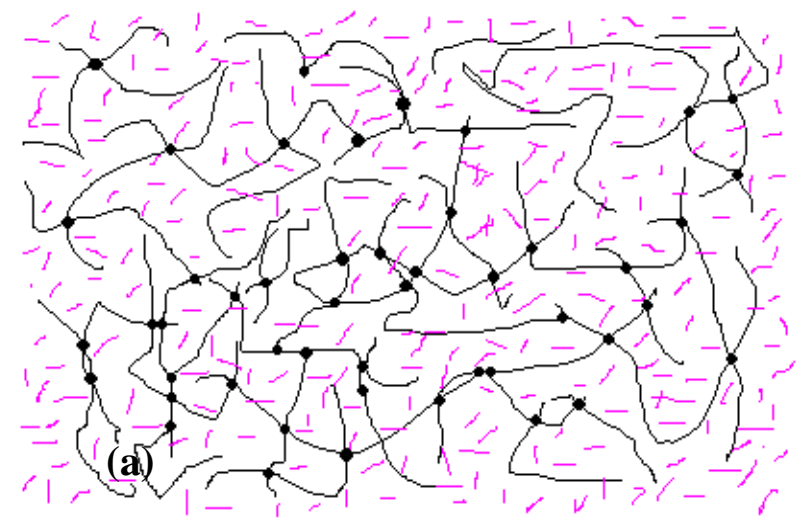

Fig. 1. (a) Uniform distribution of CNTs

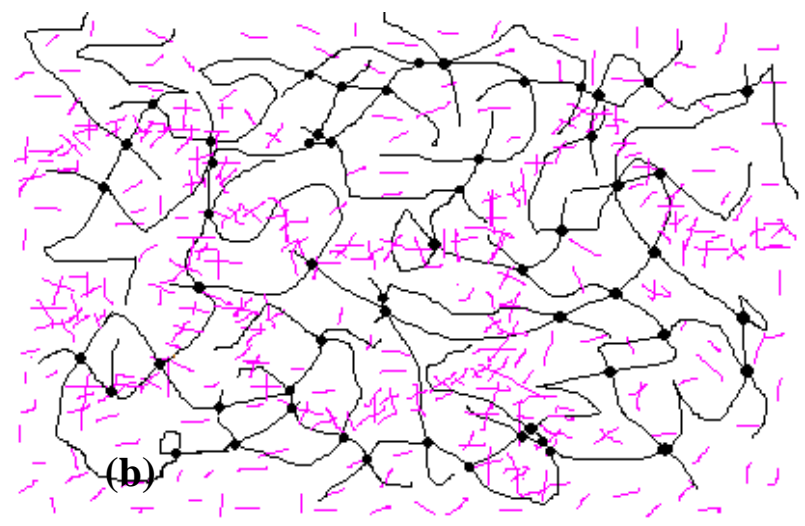

Fig. 1. (b) coexisted networks ( ER (---black) and CNTs (--pink))

This fact agrees with the FTIR findings of Wang et al.[13], where the amine groups $-\mathrm{NH}_{2}$ of the raw materials are absent after the curing while there are $\mathrm{C}-\mathrm{N}$ and $\mathrm{N}-\mathrm{H}$ bonds present in the interfacial area.

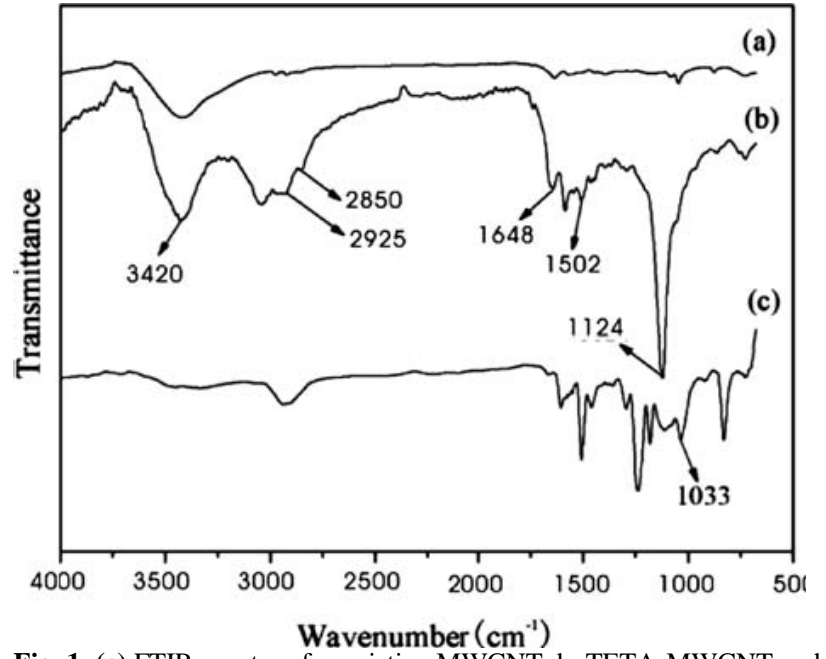

Fig. 1. (c) FTIR spectra of a. pristine MWCNT, b. TETA-MWCNT and c. TETA-MWCNT/EP [13]

These bonds are forming the secondary network which seems to affect the final properties of the product. The formation of the CNTs secondary network affects the long scale movements of the ER networks. It probably reduces the free volume and restricts the area where resin chains move.

The results indicate the existence of a stiffer interfacial layer around the filler particles and the coexistence of two networks. The discussion includes the effect of the filler content, the dispersion conditions and the architectural structure of the secondary network on the glass-transition temperature and the thermodynamic behavior of the produced material $[18,19]$

The glass transition temperature $\left(\mathrm{T}_{\mathrm{g}}\right)$ of the composites depends on the filler content. As presented in Table 2 the $T_{g}$ increases with filler content up to a maximum value corresponding to $0.1 \%$ of MWCNTs content and then it decreases slightly.

Table 2: $\mathrm{T}_{\mathrm{g}}$ values estimated with DMA and DSC methods.

\begin{tabular}{rllllll}
\hline Material Pure & Resin & Resin & Resin & Resin & Resin & Resin \\
Resin MWC & MWCNMWCNMWCNT & MWCN & MWCNTS \\
NTS & TS & TS & S 0.2\% & TS 0.3\% 0.5\% \\
$\mathbf{0 . 0 1}$ & $\mathbf{0 . 0 5 \%}$ & $\mathbf{0 . 1} \%$ & &
\end{tabular}

\begin{tabular}{llllllll}
\hline $\operatorname{Tg}\left({ }^{\circ} \mathrm{C}\right)$ & 125 & 122 & 136 & 138 & 130 & 130 & 132
\end{tabular}

(DSC)

$\begin{array}{llllllll}\operatorname{Tg}\left({ }^{\circ} \mathrm{C}\right) & 159 & 162 & 154 & 169 & 163 & 163 & 160\end{array}$

(DMA)

There are many factors which will increase the $T_{g}$ of the matrix when adding amino-functionized MWCNTs: a) The interaction between the nanotubes and the resin. The covalent bonds between the amino groups on the surface of the nanotubes and the epoxy matrix reduce the matrix mobility which express itself in shift of $\mathrm{T}_{\mathrm{g}}$ to higher values. b) The steric hindrance of the rigid segments on the aminofunctionized MWCNTs, such as benzenerings increase the inflexibility of the matrix and make epoxy resin move with difficulty, thus increasing the $\mathrm{T}_{\mathrm{g}}$ of the composites. c) The 
T. V. Kosmidou, E. Nikolaidou, A. Z. Stimoniaris, A. S. Vatalis and C. G. Delides/

Journal of Engineering Science and Technology Review 4 (2) (2011) 169-173

participation of the nanotubes to network formation. Aminofunctionized MWCNTs can be considered as a polyamide system, since it has multiple groups terminating each sidewall functionality and it is possible that more than one moiety bond to an open end of the nanotube, thus acting as another curing agent and may have effect on the initial rate on the composites. This can lead first to an epoxy-nanotube network and secondly, after a certain value of the filler content (about $0.1 \%$ ), to the formation by the nanotubes of a secondary network, as shown in figure 1b. After this point, a fully integrated network exists in the composite.

The decrease in $\mathrm{T}_{\mathrm{g}}$ may be caused by the small holes existing in the network and the formation of conglomerations. These may perform as defects increasing the free volume, which make the macromolecules to move easily, and the $\mathrm{T}_{\mathrm{g}}$ to shift to lower values.

Interesting results are also derived from DSC and TGA measurements:

The enthalpy change $(\Delta H)$ in Figure 2, calculated from the peak area of the DSC curves of curing reaction indicates a significant decrease with nanotubes content, with the maximum again at the content of $0.1 \%$.

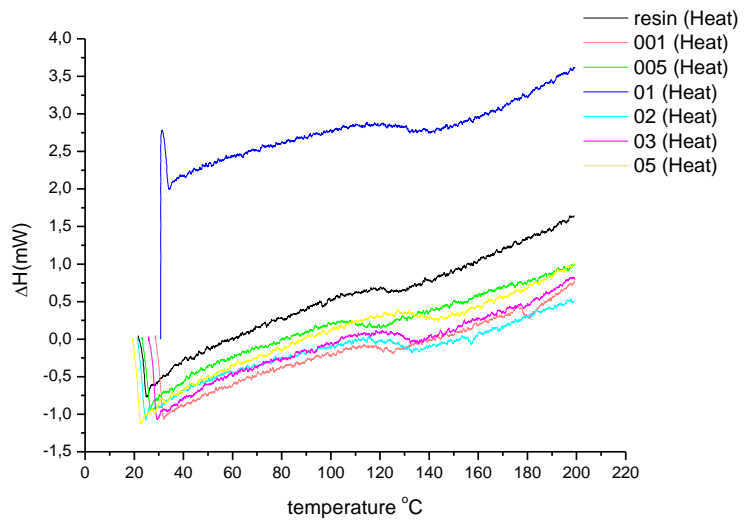

Fig. 2. DSC measurements for the system resin/MWCNTs, Dashed lines: examples of $\mathrm{T}_{\mathrm{g}}$ evaluation points.

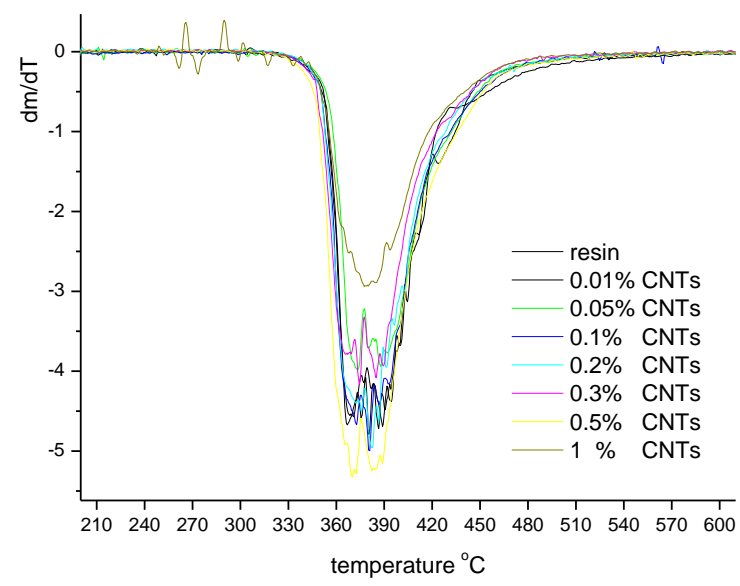

Fig. 3. TGA measurements for the systems resin/MWCNTs

In Figure 3 the results of TGA are depicted, concerning these results there are certain points of discussion. All samples start to lose weight around $300{ }^{0} \mathrm{C}$, as expected. $\mathrm{T}_{\mathrm{i}}$ temperature of the nanocomposites is slightly decreased by the addition of MWCNTs and the $\mathrm{T}_{\max }$ exhibits increased thermal stability compared to net resin. The degradation percentage is in the range between $77 \%$ and $93 \%$, while there is a large weight loss between $320-570{ }^{\circ} \mathrm{C}$ (peak at $378{ }^{0} \mathrm{C}$ ), possibly corresponding to the degradation of DGEBA/TETA system. The thermal stability parameters of nanocomposites such as the initial decomposed temperature $T_{i}$ is slightly decreased by an addition of MWCNTs. The temperature of maximum rate of weight loss $\mathrm{T}_{\max }$ of nanocomposites exhibits increased thermal stability due to the stronger linkage created by the interaction between the epoxy and the functionalized MWCNTs.

The DMA results as shown in Figure 4 reveal the following facts: The storage modulus in the glassy state is strongly affected by the amount of the MWCNTs and it shows an increase at the highest filler content, similar to the founding of Liu et al [20].

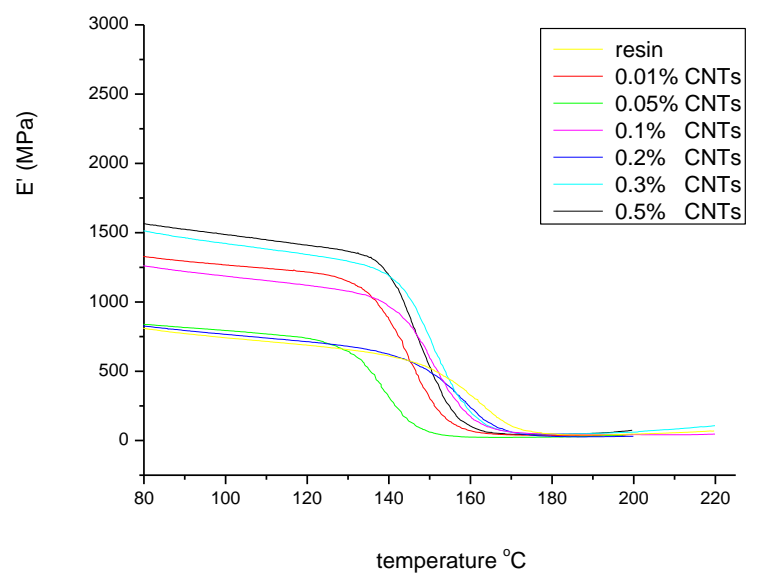

Fig. 4. DMA measurements for the system resin/MWCNTs : storage modulus

The minimum of the $\tan \delta$ height is observed at the maximum $\mathrm{T}_{\mathrm{g}}$. This is related, as mentioned before, to the "ideal" content of the MWCNTs in order to participate positively in the crosslinking. Of course it is well known that $\mathrm{T}_{\mathrm{g}}$ temperature is measured at higher values with the DMA method rather than the DSC method, because the $\alpha$ relaxation mechanism is shifted to higher temperatures with frequency (DMA measurements were performed at $10 \mathrm{~Hz}$ ). Both DSC and DMA testing involve thermal treatment of the specimens but during DMA testing the specimens are subjected to an additional 3 point bending cyclic loading from which we derive the results.

The incorporation of nanonotubes affects also the $\beta$ relaxation process, and thus the $\mathrm{T}_{\beta}$.In literature the origin of $\beta$-relaxation in polymers is explained differently: by the side groups motion, the internal vibrations and/or rotation in them, the motion of 1-2 monomeric units and the popular crankshaft mechanism which involves at least five consecutive bonds [21].The conformational change of the six-member ring on the anhydride seems to be unaffected by the filler. This is also proposed by Sun et al. [21] for epoxy composites filled with silica. The present of fillers' particles in nanocoposites adds on more factor to those mentioned above. The dependence of $\beta$-relaxation $\left(T_{\beta}\right)$ on the filler content became more complex if the filler participates to 
T. V. Kosmidou, E. Nikolaidou, A. Z. Stimoniaris, A. S. Vatalis and C. G. Delides/

Journal of Engineering Science and Technology Review 4 (2) (2011) 169-173

network formation. Thus, the understanding of the general effect of the nanotubes on the $\beta$-relaxation suggests that the complex architecture of the network affects the local-small scale motions.

The DMA spectra in the region around the $\beta$-transition temperature $\left(\mathrm{T}_{\beta}\right)$ are shown in Figure 6.

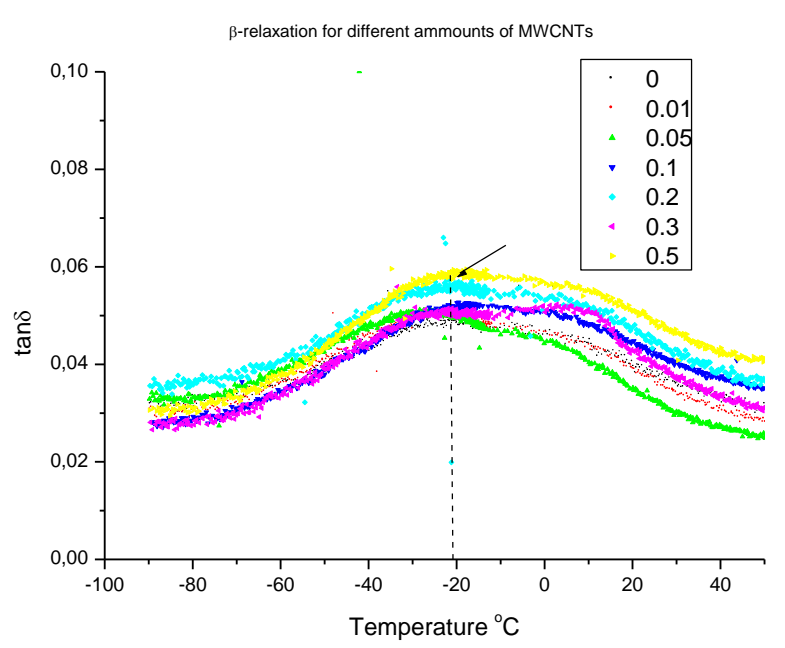

Fig. 6. DMA measurements for the system resin/MWCNTs: $\beta$ relaxation. Example of $\mathrm{T}_{\beta}$ point evaluation: arrow and dash line.

The temperature amplitude of the relaxation became broader and $\mathrm{T}_{\beta}$ values are shifted to higher temperatures. Locomotion to higher temperatures occurs due to the functionalization of the MWCNTS which contributes to the greater stiffness of the interface and restricts even the smaller segments movements in the material.

\section{Conclusions}

The addition of the amine modified MWCNTs in the epoxy matrix results in a system that exhibits different properties than the pure bulk matrix, and/or the matrix reinforced with non-modified nanotubes, mainly because of their participation to the crosslinking procedure.

The functionalization of the MWCNTs contributes to the formation of a network with complex architecture through the improvement and extension of the interface layer in a way that the pure unaffected matrix regions in the system are absent.

The formation of the CNTs secondary network, which is completed at a certain value of the filler content coexisted with some agglomerated areas, affects both the long and the short scale movements ( $\alpha$ - and $\beta$-relaxations) of the ER networks. Above certain critical content of MWCNTs, which is between 0.05 and $0.1 \% \mathrm{w} / \mathrm{w}$, - the glass transition temperature $\left(\mathrm{T}_{\mathrm{g}}\right)$ is higher than that of the pure bulk matrix due to the significant improvement of the interfacial bonding, which lead to a reduction of the epoxy matrix mobility

The glass transition temperature of the composites depends on the filler content. $\mathrm{T}_{\mathrm{g}}$ increases up to a maximum corresponding to a critical value of about $0.1 \% \mathrm{w} / \mathrm{w}$ of the MWCNTs content and then decreases again. The position of the $T_{\beta}$ (sub- $T_{g}$ ) transition is slightly shifted to higher temperatures with filler content.

\section{Acknowledgements}

The authors would like to thank Prof. Richard Pethrick of Strathclyde University for his valuable discussion and the students C. Liakopoulos and M-A Kardasi for their help to the sample preparation.

\section{References}

1. A.W. Musumeci, G.G. Silva, W.N. Martens, E.R. Waclawik, R.L. Frost, Journal of Thermal Analysis and Calorimetry 88 885-891 (2007)

2. J.P.C. Trigueiro, G.G. Silva, R.L. Lavall, C.A. Furtado, S. Oliveira, A.S. Ferlauto, R.G. Lacerda, L.O. Ladeira, J.-W. Liu, R.L. Frost, G.A. George, Journal of Nanoscience and Nanotechnology 73477 3486 (2007).

3. Yangyang S., Zhuoing Z.,Kyoung-sik M.,Wong C.P, J Polym Sci Part B: Polym Phys 42, 3849-3858 (2004)

4. Brown J., Rhoney I.,.Pethrick R.A, Polymer International 53 (12) 2130 (2004)

5. CaoY. M., Sun, J., Yu D. H., J Appl Polym Sci 83, 70 (2002).

6. Pham, J. Q.; Mitchell, C. A.; Bahr, J. L.; Tour, J. M.;Krishanamoorti, R.; Green, P. F. J Polym Sci PartB: Polym Phys 41, 3339 (2003).

7. Ash, B. J.; Schadler, L. S.; Siegel, R. W. Mater Lett 55, 83 (2002).

8. Xiong, M.; Gu, G.; You, B.; Wu, L. J Appl Polym Sci 90, 1923 (2003).

9. R. Kotsilkova, D. Fragiadakis and P. Pissis J Polym Sci Part B: Polym Phys 43, 522-533 (2005).

10. B. Dong, Z. Yang, Y. Huang and H.-L. Li, Tribology Letters, Vol. 20, 251-254 (2005)
11. T. P. Mohan, M. Ramesh Kumar, R. Velmurugan, J Mater Sci 41 2929-2937 (2006)

12. Smrutisikha Bal, Bulletin of Material Science, Springer, vol.33, no.1, February 2010, pp. 1-5

13. Fang Wang $Æ$ Jing-Wen Wang $Æ$ Shu-qin Li $Æ$ Jun Xiao, Polym. Bull 63:101-110. (2009)

14. L. E. Evseeva and S. A. Tanaeva, Me chanics of Com pos ite Ma te rials, Vol. 44, No. 5, (2008)

15. Zdenko Spitalský, Libor Matějka , Miroslav Slouf, Elena N. Konyushenko, Jana Kováł ová, Josef Zemek, Jił í Kotek, Polymer Composites, 30, 1378-1387 (2009)

16. Woo Jin Choi, Robert L. Powell, Dae Su Kim Polymer Composites, 30, 415-421 (2009)

17. Berriot J., Montes H., Lequeux F., Long D.,Sotta P. Macromolecules 35, 9756 (2002).

18. Ming-Jen Chang, Lin-Fen Chang, George J. Jiang, POLYMER COMPOSITES, 2007, 390-39

19. M. Chen, A.S. Ren, J.F. Wang, M.S Lee, L.R Dalton H. Zhang, G. Sun, and W.H. Steirer. Polym. Prepr. 40162 (1999)

20. Liu M., Guo, B., Du M., J Polym Res 15, 205-212 (2008).

21. Y. Sun, Z. Zhang, K-S. Moon, C. P. Wong, J Polym Sci 42, 38493858 (2004). 